IJMMS 27:11 (2001) 695-699

PII. S0161171201010547

http://ijmms.hindawi.com

(C) Hindawi Publishing Corp.

\title{
STRICTLY WEBBED SPACES AND REGULARITY PROPERTIES OF INDUCTIVE LIMITS
}

\author{
ARMANDO GARCÍA-MARTÍNEZ
}

(Received 9 January 2001)

\begin{abstract}
Sequentially complete, locally complete, locally Baire, and bornivorously webbed are equivalent for strictly webbed spaces. For inductive limits of strictly webbed spaces these properties are equivalent. Moreover, they imply regularity.
\end{abstract}

2000 Mathematics Subject Classification. 46A13, 46A17.

1. Introduction. Throughout this note $E$ is a locally convex space and $E_{1} \subset E_{2} \subset \cdots$ is a sequence of Hausdorff locally convex spaces with continuous identity maps id : $\left(E_{n}, \tau_{n}\right) \rightarrow\left(E_{n+1}, \tau_{n+1}\right), n \in \mathbb{N}$ where $\tau_{n}$ is the topology of $E_{n}$. Their locally convex inductive limit is denoted by ind $E_{n}$. A web $W$ in a locally convex space $E$ is a countable family of absolutely convex subsets of $E$, arranged in layers. The first layer of the web consists of a sequence $\left(A_{p}: p=1,2, \ldots\right)$ whose union absorbs each point of $E$. For each set $A_{p}$ of the first layer there is a sequence $\left(A_{p q}: q=1,2, \ldots\right)$ of sets, called the sequence determined by $A_{p}$, such that

$$
\begin{gathered}
A_{p q}+A_{p q} \subset A_{p} \text { for each } q, \\
\bigcup\left\{A_{p q}: q=1,2, \ldots\right\} \quad \text { absorbs each point of } A_{p} .
\end{gathered}
$$

Further layers are made up in a corresponding way so that each set of the $k$ th layer is indexed by a finite row of $k$ integers and at each step the above mentioned two conditions are satisfied. Suppose that we choose a set $A_{p}$ from the first layer, then a set $A_{p q}$ of the sequence determined by $A_{p}$ and so on. The resulting sequence $S=\left(A_{p}, A_{p q}, A_{p q r}, \ldots\right)$ is called a strand. Whenever we are dealing with only one strand we can simplify the notation by writing $W_{1}=A_{p}, W_{2}=A_{p q}$, and so forth, thus $S=\left(W_{k}\right)$ is a strand where for each $k, W_{k}$ is a set of the $k$ th layer.

Let $S=\left(W_{k}\right)$ be a strand. Consider $x_{k} \in W_{k}$ and the series $\sum_{k=1}^{\infty} x_{k}$. The space $E$ is webbed if the series $\sum_{k=1}^{\infty} x_{k}$ is convergent for any choice of $x_{k} \in W_{k}$; $E$ is strictly webbed if $\sum_{k=n+1}^{\infty} x_{k}$ converges to some element in $W_{n}$ for every $n \in \mathbb{N}$ and for any choice of $x_{k} \in W_{k}$;E is bornivorously webbed if it is strictly webbed and for every bounded set $A \subset E$, there exist a strand $\left(W_{k}\right)_{k}$ and a sequence $\left(\alpha_{k}\right)_{k} \subset \mathbb{C}$ such that $A \subset \alpha_{k} W_{k}$, for every $k \in \mathbb{N}[2,6,7,9]$.

A disk $A \subset E$ is an absolutely convex, bounded and closed set. Let $E_{A}$ denote the linear span of $A$ endowed with the normed topology generated by the Minkowski functional $\rho$ of $A$. This topology is finer than the topology inherited from $E$. If $\left(E_{A}, \rho_{A}\right)$ is a Banach (Baire) space, $A$ is a Banach (Baire) disk. A locally convex space is locally 
complete (locally Baire) if every bounded subset is contained in a Banach (Baire) disk. $E$ is a quasi-locally complete space if for each bounded subset $B$ in $(E, \tau)$ there exists a weaker locally convex topology $\varsigma=\varsigma(B)$ on $E$ and a Banach disk $A$ in $(E, \varsigma)$ such that $B \subset A$ [8]. Note that locally complete implies quasi-locally complete.

$E$ satisfies the Mackey convergence condition if for every null sequence $\left(x_{n}\right)_{n} \subset E$, there exists a disk $A$ such that $\left(x_{n}\right)_{n}$ is a $\rho_{A}$-null sequence. Finally, $E$ satisfies property $K$ if each null sequence has a series convergent subsequence.

\section{Bornivorously webbed}

LEMMA 2.1. Let $(E, \tau)$ be a bornivorously webbed space. Then for every bounded set $A \subset E$ there exists a Fréchet space $(F, \gamma)$ such that $A$ is contained and bounded in $F$.

Proof. Let $A \subset E$ be a bounded set. Then there exist a strand $\left(W_{k}\right)_{k} \subset W$ and a sequence $\left(\alpha_{k}\right)_{k} \subset \mathbb{C}$ such that $A \subset \alpha_{k} W_{k}$, for every $k \in \mathbb{N}$. Consider $E_{W_{k}}=\operatorname{span}\left(W_{k}\right)$ and $F=\bigcap_{k \in \mathbb{N}} E_{W_{k}}$. Let $\left\{F \cap(1 / k) W_{k}: k \in \mathbb{N}\right\}$ be a fundamental system of neighborhoods of zero in $F$. This topology is metrizable and finer than $\tau$. We will see that it is complete. Let $\left(x_{k}\right)_{k} \subset F$ be a Cauchy sequence, and take $\left(y_{k}\right)_{k} \subset\left(x_{k}\right)_{k}$ such that $\left(y_{k+1}-y_{k}\right) \in$ $W_{k} / k$. Then $\sum_{k=1}^{\infty}\left(y_{k+1}-y_{k}\right) \stackrel{\tau}{\rightarrow} u$, for some $u$ in $E$. $\sum_{k=p+1}^{\infty}\left(y_{k+1}-y_{k}\right) \in W_{p} / p$, for every $p \in \mathbb{N}$, so $\sum_{k=1}^{\infty}\left(y_{k+1}-y_{k}\right) \in F$. Hence $\sum_{k=1}^{\infty}\left(y_{k+1}-y_{k}\right) \stackrel{F}{\rightarrow} u$ and if $x=u+y_{1}$, we have $y_{k} \stackrel{F}{\rightarrow} x$ and $x_{k} \stackrel{F}{\rightarrow} x$.

If $F=E$, and $\left\{(1 / k) W_{k}: k \in \mathbb{N}\right\}$ is a fundamental system of neighborhoods, then $E$ with the topology $\gamma$ generated by this family is a Fréchet space. This topology is finer than the original one.

THEOREM 2.2. Let $(E, \tau)$ be a locally convex space. If $E$ is strictly webbed, then the following properties are equivalent:

(a) $E$ is sequentially complete.

(b) $E$ is locally complete.

(c) $E$ is locally Baire.

(d) $E$ is bornivorously webbed.

Proof. (a) $\Rightarrow(\mathrm{b}) \Rightarrow$ (c). The proof is obvious. (c) $\Rightarrow(\mathrm{d})$. Let $A$ be a bounded subset of $E$ and $B \subset E$ be a Baire disk such that $A$ is contained and bounded in $B$. By [6, Theorem 5.6.3] for id : $E_{B} \rightarrow E$, there exists a strand $\left(W_{k}\right)_{k}$ such that $\operatorname{id}^{-1}\left(W_{k}\right) \in N_{0}\left(E_{B}\right)$. Hence, for every $k \in \mathbb{N}$ there exists $\alpha_{k} \in \mathbb{C}$ such that $A \subset \alpha_{k} \mathrm{id}^{-1}\left(W_{k}\right) \subset E_{B}$ and $A \subset$ $\alpha_{k} W_{k} \subset E$.

(d) $\Rightarrow(\mathrm{a})$. The argument of the proof is taken from [1, Theorem 1]: let $\left(x_{n}\right)_{n}$ be a Cauchy sequence in $E$, and $B_{n}=\operatorname{cl}_{E} \operatorname{co\bigcup }\left\{x_{n}: m \geq n\right\}, n \in \mathbb{N}$. The set $B_{1}$ is bounded in $E$ which is bornivorously webbed, hence there exists a strand $\left(W_{k}\right)$ in $E$ and a sequence $\left(\alpha_{k}\right)_{k} \subset \mathbb{C}$ such that $B_{1} \subset \alpha_{k} W_{k}$ for each $k \in \mathbb{N}$. Denote by $\gamma$ the topology on $E$ generated by the subbasis $\left\{W_{k}: k \in \mathbb{N}\right\}$ and, for brevity, by $F$ the space $(E, \gamma)$.

The set $B_{1} \subset E$ is closed in $E$, and by the preceding lemma, it is closed in the locally convex space $F$. Since $B_{1}$ is convex, it is also weakly closed in $F$.

By lemma, $F$ is a Fréchet space. Hence the canonical imbedding $F \rightarrow F^{\prime \prime}$, where $F^{\prime \prime}$ is the second dual of $F$ equipped with the strong topology, is a topological isomorphism 
into $F^{\prime \prime}$. Since $F$ is complete, it is closed in $F^{\prime \prime}$ and each functional from the strong dual $F^{\prime}$ of $F$ can be continuously extended to $F^{\prime \prime}$. Thus the $\sigma\left(F, F^{\prime}\right)$-closed set $B_{1}$ is also $\sigma\left(F^{\prime \prime}, F^{\prime}\right)$-closed in $F^{\prime \prime}$.

Further, since $B_{1}$ is bounded in $F^{\prime \prime}$, it is equicontinuous in $F^{\prime}$. Hence by Alaoglu theorem, the set $B_{1}$ is relatively $\sigma\left(F^{\prime \prime}, F^{\prime}\right)$-compact. This, together with the $\sigma\left(F^{\prime \prime}, F^{\prime}\right)$ closedness, implies that $B_{1}$ is $\sigma\left(F^{\prime \prime}, F^{\prime}\right)$-compact in $F^{\prime \prime}$.

Similarly, all sets $B_{n}, n \in \mathbb{N}$, are $\sigma\left(F^{\prime \prime}, F^{\prime}\right)$-compact. Every finite intersection $\bigcap\left\{B_{n}\right.$ : $1 \leq n \leq m\}=B_{m}, m \in \mathbb{N}$, is nonempty. Hence there exists $x_{0} \in \bigcap\left\{B_{n}: n \in \mathbb{N}\right\} \subset B_{1} \subset E$. This implies the existence of an upper triangular matrix $\Lambda=\left(\lambda_{n m}\right)$ with all entries $\lambda_{n m} \geq 0$, only finite number of nonzeros in each row, and the sum of all entries in each row is equal to 1 , such that the sequence $\left\{y_{n}=\sum_{m=n}^{\infty} \lambda_{n m} x_{m}\right\}_{n}$ converges to $x_{0}$ in the topology $\gamma$. Then the continuity of the identity map $F \rightarrow E$ implies the convergence $y_{k} \rightarrow x_{0}$ in $E$.

Take a balanced, convex, zero neighborhood $V$ in $E$. Then there exist $p, q \in \mathbb{N}$ such that $y_{n}-x_{0} \in V$ for $n \geq p$ and $x_{m}-x_{n} \in V$ for $m \geq n \geq q$. Then for $n \geq \max (p, q)$, we have

$$
x_{0}-x_{n}=\left(x_{0}-y_{n}\right)+\left(y_{n}-x_{n}\right)=\left(x_{0}-y_{n}\right)+\sum_{m=n}^{\infty} \lambda_{n m}\left(x_{m}-x_{n}\right) \in V+V .
$$

This implies $x_{n} \rightarrow x_{0}$ in the space $E$.

Since property $K$ implies locally Baire (see [4, Theorem 2]), this theorem proves that for strictly webbed spaces, property $K$ implies local completeness. This answers Gilsdorf's question 3.2 in [4] in a negative way. Moreover, these different additional properties for strictly webbed spaces, which appear in $[1,4,5]$, are proved to be all equivalent.

3. Inductive limits. Let $\left(E_{n}, T_{n}\right)_{n}$ be an inductive sequence of locally convex spaces, and let $(E, \tau)=\operatorname{ind}\left(E_{n}, \tau_{n}\right)$ be its inductive limit. The space $(E, \tau)$ is regular if for each bounded subset $B$ in $(E, \tau)$, there exists $n=n(B) \in \mathbb{N}$ such that $B$ is contained and bounded in $\left(E_{n}, \tau_{n}\right) .(E, \tau)$ is sequentially retractive if for each convergent sequence $\left(x_{k}\right)_{k}$ in $(E, \tau)$ there exists $n=n\left(\left(x_{k}\right)_{k}\right) \in \mathbb{N}$ such that the sequence converges to the same limit in $\left(E_{n}, \tau_{n}\right)$. Equivalently, each null sequence in $(E, \tau)$ is a null sequence in some $\left(E_{n}, \tau_{n}\right)$.

Sequentially retractive inductive limits were introduced and studied by Floret [3, 7]. He proved that sequential retractivity implies regularity. In order to get more information about the relation between regularity and sequential retractivity, we will prove the following proposition.

Proposition 3.1. Let $(E, \tau)=\operatorname{ind}\left(E_{n}, \tau_{n}\right)$ be a regular inductive limit. If $E$ satisfies the Mackey convergence condition, then it is sequentially retractive.

Proof. Let $\left(x_{k}\right)_{k}$ be a null sequence in $E$. Since the Mackey convergence condition holds, there exists a bounded disk $B \subset E$ such that $\left(x_{k}\right)_{k}$ is a $\rho_{B}$-null sequence. Now $E$ is regular, so $B$ is contained and bounded in some $E_{n}$. So, the topology $\rho_{B}$ in $E_{B} \subset E_{n}$, is finer than that inherited from $E_{n}$. Hence $\left(x_{k}\right)_{k}$ is an $E_{n}$-null sequence. 
In the next propositions, we present other relations between these properties and regularity for strictly webbed spaces.

Following Floret [3] and the proof of Theorem 1 in [1], if $(E, \tau)=\operatorname{ind}\left(E_{n}, \tau_{n}\right)$ is an inductive limit of an inductive sequence of bornivorously webbed spaces note that we have:

$E$ sequentially retractive it follows that $E$ is regular and implies that if $x_{k} \stackrel{\tau}{\rightarrow} x_{0}$, then there exists $n_{0} \in \mathbb{N}$ and a sequence $\left\{y_{k}: y_{k} \in \operatorname{conv}\left\{x_{m}\right\}_{m=k}^{\infty}\right\}_{k=1}^{\infty}$ such that $y_{k} \stackrel{T_{n_{0}}}{\longrightarrow} x_{0}$.

THEOREM 3.2. Let $(E, \tau)=\operatorname{ind}\left(E_{n}, \tau_{n}\right)$ be the inductive limit of an inductive sequence of strictly webbed locally convex spaces. Consider the conditions:

(a) E satisfies property $K$

(b) $E$ is locally Baire

(c) E is bornivorously webbed

(d) E is sequentially complete

(e) E is locally complete

(f) E is quasi-locally complete

(g) $E$ is regular.

Then $(\mathrm{a}) \Rightarrow(\mathrm{b}) \Leftrightarrow(\mathrm{c}) \Leftrightarrow(\mathrm{d}) \Leftrightarrow(\mathrm{e})$ and $(\mathrm{e}) \Rightarrow(\mathrm{f}) \Rightarrow(\mathrm{g})$.

Proof. (a) $\Rightarrow(\mathrm{b})$. [4, Theorem 2].

(b) $\Leftrightarrow(\mathrm{c}) \Leftrightarrow(\mathrm{d}) \Leftrightarrow(\mathrm{e})$. By Theorem 2.2, since the inductive limit of strictly webbed spaces is strictly webbed.

(e) $\Rightarrow(\mathrm{f})$. It is clear.

$(\mathrm{f}) \Rightarrow(\mathrm{g})$. [8, Theorem 1].

Proposition 3.3. Let $(E, \tau)=\operatorname{ind}\left(E_{n}, \tau_{n}\right)$ be the inductive limit of an inductive sequence of strictly webbed locally convex spaces such that every $\left(E_{n}, \tau_{n}\right)$ satisfies property $K$. If $E$ is sequentially retractive then $E$ satisfies property $K$.

Proof. Let $\left(x_{m}\right)_{m}$ be a null sequence in $E$. Then there exist $n \in \mathbb{N}$, with $x_{m} \stackrel{E_{n}}{\longrightarrow}$ 0 and a subsequence $\left(x_{m_{k}}\right)_{k} \subset\left(x_{m}\right)_{m}$ such that $\sum_{k=1}^{\infty} x_{m_{k}} \stackrel{E_{n}}{\longrightarrow} x$. Therefore $\sum_{k=1}^{\infty}$ $x_{m_{k}} \stackrel{E}{\longrightarrow} x$.

Note that combining the results of this section, and under the hypothesis of Proposition 3.3 we have $(\mathrm{a}) \Rightarrow(\mathrm{b}) \Leftrightarrow(\mathrm{c}) \Leftrightarrow(\mathrm{d}) \Leftrightarrow(\mathrm{e}) \Rightarrow(\mathrm{f}) \Rightarrow(\mathrm{g})$. Moreover if $E$ satisfies the Mackey convergence condition they are all equivalent.

ACKNowledgement. I would like to thank Dr. C. Bosch for his valuable suggestions.

\section{REFERENCES}

[1] C. Bosch and J. Kucera, Sequential completeness and regularity of inductive limits of webbed spaces, to appear in Czechoslovak Math. J.

[2] M. de Wilde, Closed Graph Theorems and Webbed Spaces, Research Notes in Mathematics, vol. 19, Pitman, Massachusetts, 1978. MR 81j:46013. Zbl 373.46007.

[3] K. Floret, Some aspects of the theory of locally convex inductive limits, Functional Analysis: Surveys and Recent Results, II (Proc. Second Conf. Functional Anal., Univ. Paderborn, Paderborn, 1979), North-Holland Math. Stud., vol. 38, North-Holland, Amsterdam, 1980, pp. 205-237. MR 81j:46009. Zbl 461.46002. 
[4] T. E. Gilsdorf, Regular inductive limits of K-spaces, Collect. Math. 42 (1991), no. 1, 45-49. MR 93i:46011. Zbl 772.46001.

[5] _ Boundedly compatible webs and strict Mackey convergence, Math. Nachr. 159 (1992), 139-147. MR 94g:46004. Zbl 808.46006.

[6] H. Jarchow, Locally Convex Spaces. Mathematische Leitfaden. [Mathematical Textbooks], B. G. Teubner, Stuttgart, 1981. MR 83h:46008. Zbl 466.46001.

[7] G. Köthe, Topological Vector Spaces. II, Grundlehren der mathematischen Wissenschaften, vol. 237, Springer-Verlag, New York, 1979. MR 81g:46001. Zbl 0417.46001.

[8] J. Qiu, Quasi-fast completeness and inductive limits of webbed spaces, J. Math. Res. Exposition 18 (1998), no. 1, 55-59. MR 99d:46003. Zbl 926.46002.

[9] W. Robertson, On the closed graph theorem and spaces with webs, Proc. London Math. Soc. (3) 24 (1972), 692-738. MR 46\#5979. Zbl 238.46005.

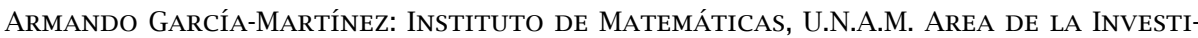
gaCión Científica, Circuito Exterior, Ciudad Universitaria, MÉXico, D.F. 04510, MeXico E-mail address: agarcia@matem. unam.mx 


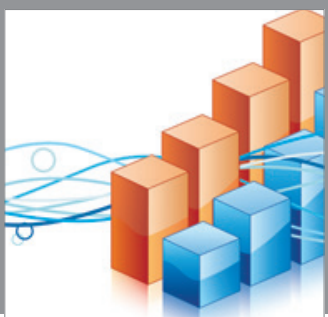

Advances in

Operations Research

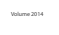

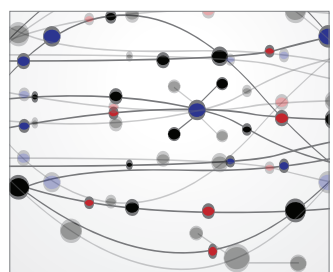

\section{The Scientific} World Journal
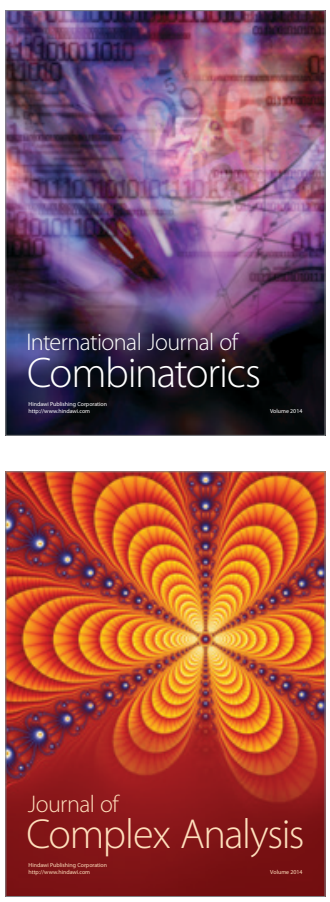

International Journal of

Mathematics and

Mathematical

Sciences
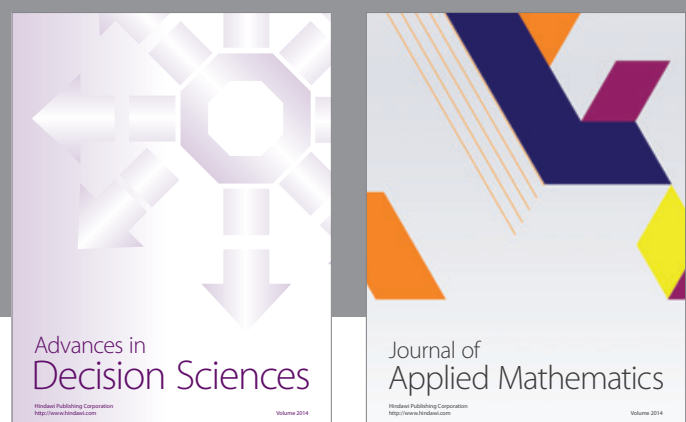

Journal of

Applied Mathematics
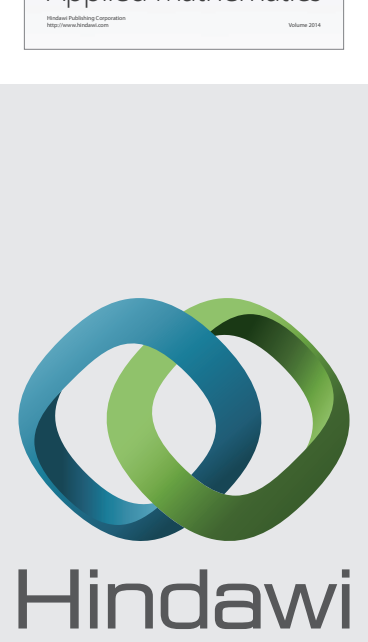

Submit your manuscripts at http://www.hindawi.com
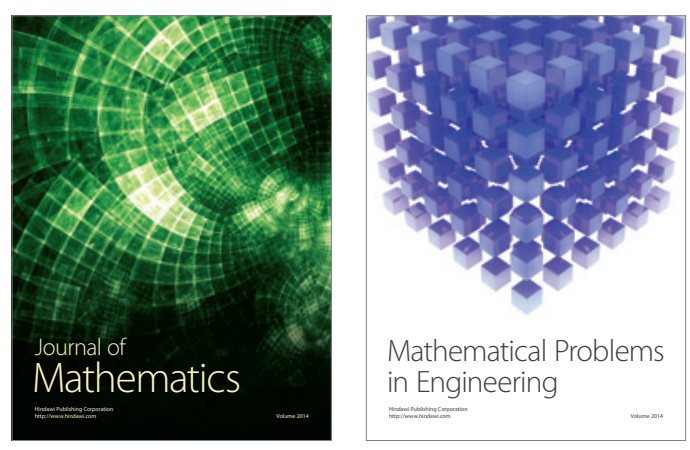

Mathematical Problems in Engineering
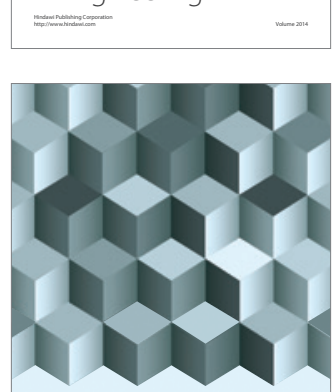

Journal of

Function Spaces
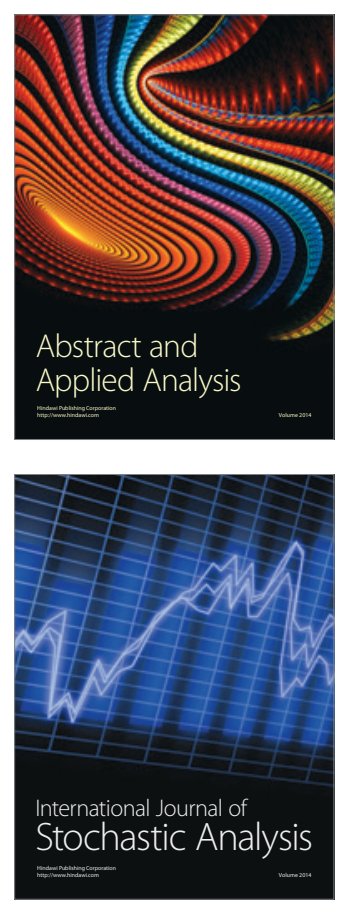

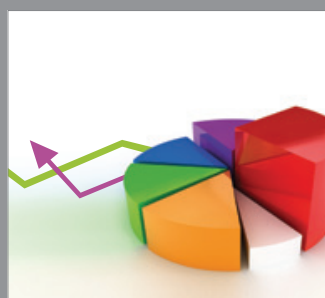

ournal of

Probability and Statistics

Promensencen
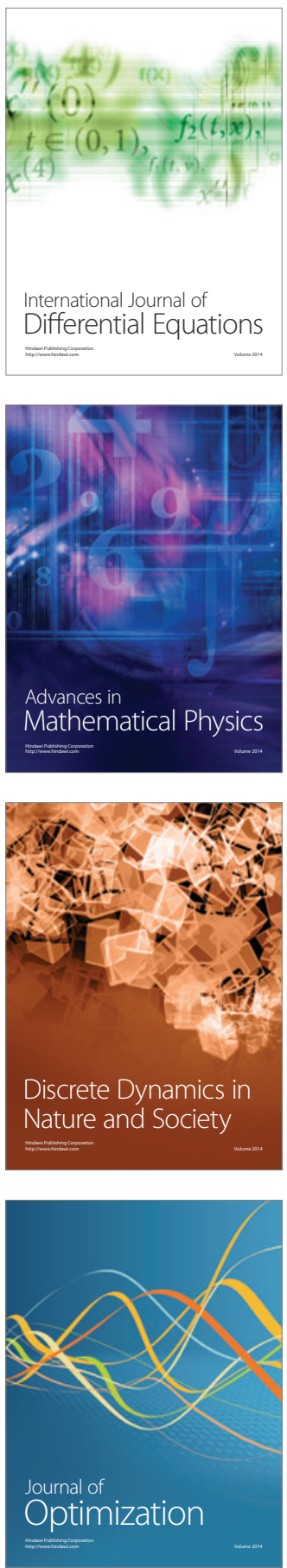